\title{
8
}

\section{Thermophysical Properties of Bi-based High-Tc Superconductors}

\author{
Asghari Maqsood ${ }^{1}$ and M. Anis-ur-Rehman ${ }^{2}$ \\ 1Thermal Transport Laboratory, School of Chemical and Materials Engineering, \\ National University of Sciences and Technology (NUST), \\ ${ }^{2}$ Applied Thermal Physics Laboratory, Department of Physics, \\ COMSATS Institute of Information Technology, \\ Islamabad, \\ Pakistan
}

\section{Introduction}

Since the discovery of $90 \mathrm{~K}$ superconductivity in the Ba-Y-Cu oxide system (Wu, et.al., 1987) a number of studies have been published. A true superconductor not only shows zero resistance but also excludes a magnetic field completely (the Meissner effect). A visual demonstration of the Meissner effect was carried out by placing a small magnet on a pellet of $\mathrm{Dy}_{1} \mathrm{Ba}_{2} \mathrm{Cu}_{3} \mathrm{O}_{7-\mathrm{x}}$ and cooling the system to liquid-nitrogen temperature. The levitation of the magnet due to ejection of magnetic lines of flux from the superconductor is shown in Figure 1 (Maqsood, et.al., 1989 ).

Dissipation phenomena in high temperature superconductors are governed by the microstructure that develops during the preparation process. Therefore, detailed investigations of the electrical and thermal transport and ac magnetic susceptibilities in

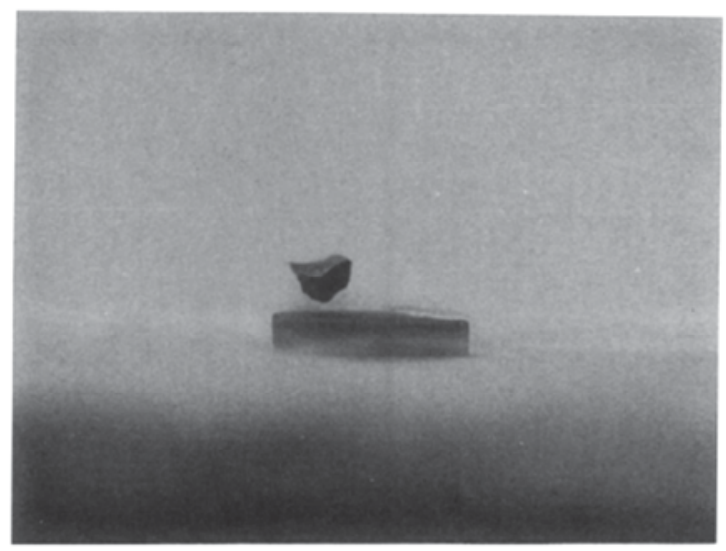

Fig. 1. The $\mathrm{Dy}_{1} \mathrm{Ba}_{2} \mathrm{Cu}_{3} \mathrm{O}_{7}-\mathrm{x}$ specimen, showing the Meissner effect at liquid-nitrogen temperature. 
superconductors prepared either in the form of single crystals, thin films or polycrystalline are important for understanding superconductivity as well as for practical applications (Rehman \& Maqsood, 2005).

Among high- $\mathrm{T}_{\mathrm{c}}$ superconductors, $(\mathrm{Bi}, \mathrm{Pb})-2223$ appears to be the most promising candidate for the application of power transmission cables at liquid nitrogen temperature. Unlike other high-Tc superconductors (HTS), such as $\mathrm{YBa}_{2} \mathrm{Cu}_{3} \mathrm{O}_{7-\delta}(\mathrm{Y}-123)$, it is still a problem to control and increase its critical temperature and current density. The Bi based superconductors offer potential advantages in comparison to the Y-based superconductors. The studies of transport properties, such as electrical resistivity, thermoelectric power (S) and thermal conductivity, are important for exploring the conduction mechanisms. The transport properties are very sensitive to the sample preparation methods.

The BISCCO samples substituted with Fe, Cr, Co, Gd, Er, Nd, Sm, Ag, V, Ga, Zn, Cd, etc. have been widely prepared using conventional solid state reaction and glass-ceramics techniques (Aksan \& Yakyncy, 2004; Chatterjee, et.al. 1998; Cloots, et.al., 1994; Coskun, et.al. 2005; Dorbolo, et.al. 1999; Ekicibil, et.al., 2004, 2005; Mandal, et.al., 1992; Munakata, et.al., 1992; Nanda, et.al., 1995; Ozhanli,et.al., 2002; Rao,et.al. 1990; Sera,et.al. 1992; Varoy, et.al. 1992). Investigation of thermal conductivity, $\lambda(\mathrm{T})$, also gives important information about the scattering mechanism of charge carriers, electron-phonon interaction and other physical properties, such as carrier density and phonon mean free path (Aksan, et.al. 1999; Houssa \& Ausloos, 1996; Knizek, et.al. 1998; Natividad,et.al. 2002; Uher,et.al. 1994; Yankyncy 1997). In the last decade, many investigations have been made on $\lambda(\mathrm{T})$ of high- $\mathrm{T}_{\mathrm{c}}$ materials (Aksan, et.al. 1999; Castellazzi, et. al. 1997; Houssa, et.al. 1996; Hui, et.al. 1999; Knizek, et.al. 1998; Natividad,et.al. 2002; Uher,et.al. 1994; Yankyncy 1997; Wermbter, 1991) and almost similar results are reported. In general, for $\lambda(\mathrm{T})$ investigation of high- $\mathrm{T}_{\mathrm{c}}$ materials, three important approaches can be considered to the total $\lambda(\mathrm{T})$ calculations: (i) phonon contribution; (ii) electron contribution; and (iii) both electron and phonon contributions. Many research groups have investigated these valuable approaches for high- $\mathrm{T}_{\mathrm{c}}$ materials and results are published (Castellazzi, et.al., 1997; Peacor, et.al., 1991; Tewordt \& Wolkhausen, 1989,1990; Wermbter, et.al., 1996; Yu, et.al. 1992). However, there exists a difficulty in the $\lambda(\mathrm{T})$ properties of the high- $\mathrm{T}_{\mathrm{c}}$ materials. In particular, compared with conventional metallic structures, the high- $\mathrm{T}_{\mathrm{c}}$ superconductors show unusual behavior just below their $\mathrm{T}_{\mathrm{c}}$. At that point, thermal conductivity rises and reaches to the maximum and then drops sharply. The explanation of the rapid rise and the maximum point seen in a wide range just below $T_{c}$, is summed up through two main points (Uher, et.al., 1994). Firstly, decrease on the scattering mechanism, because of the superconducting state $\left(T<T_{c}(R=0)\right.$, and secondly, an increase in the electron mean free path due to decrease in the phonon scattering. In many investigations, the maximal value was also found to depend on the preparation method and chemical composition (Cohn, et.al. 1992; Jezowski,et.al. 1987; Morelli,et.al. 1987; Peacor, et.al. 1991; Uher 1992; ). However, it is important to see the effect of the quasi-particle contribution on the rapid rise of $\lambda(\mathrm{T})$ below the $\mathrm{T}_{\mathrm{c}}$, as explained by many groups (Castellazzi, et.al. 1997; Yu, et.al. 1992). There exist some other models that have been widely accepted for materials in solid state. Particularly, for the graded materials, effective medium approximation (EMA) (Hirai, 1996; Hui, et.al. 1999) and another model developed for the conventional low-Tc superconductors by Bardeen et al (Bardeen, et.al., 1959) that describes the phonon thermal conductivity in the superconducting state. This model then was generalized by Tewordt and Wolkhausen in order to describe the phonon thermal 
conductivity of high- $\mathrm{T}_{\mathrm{c}}$ superconductors in a wide range of temperatures (Tewordt \& Wolkhuasen, 1989). However, still many efforts have to be made both experimentally and theoretically to understand the $\lambda(\mathrm{T})$ mechanism of high- $\mathrm{T}_{\mathrm{c}}$ materials.

Thermoelectric power being sensitive to the energy dependence of the electron lifetime and the density of states near the Fermi level energy, provides valuable information regarding many fundamental aspects of charge carrier transport in the materials. The thermoelectric power (S) of high-temperature superconductors has been widely studied and reported a positive ' $S$ ', while Khim et al. (Khim et. al. 1987) and others have reported a negative ' $S$ ' for the same compositions. Later studies proved that the sign of the thermoelectric power is sensitive to the oxygen content present in the compound (Lee, et. al. 1988). This behavior was also observed in other materials like Cheverly phase compounds as was seen by Vasudeva Rao et al. (Rao, et.al. 1984). In the BISCCO compounds, the TEP studies earlier reported on the (2201), (2212) and (2223) phases. Sera et al. (Sera et. al. 1992) have studied the $S$ behavior of La doped (2201) cuprates. The ' $S$ ' behavior of the $\mathrm{Bi}_{2} \mathrm{Sr}_{2} \mathrm{Ca}_{1-x} \mathrm{Y}_{x} \mathrm{Cu}_{2} \mathrm{O}_{y}$ compounds was investigated as a function of temperature (Mandal, et.al. 1992; Munakata, et.al. 1992; Varoy, et. al. 1992) and it was found that with increasing temperature ' $S$ ' increases, exhibits a maximum value and decreases thereafter. From the ' $S$ ' data they concluded that the substitution of $\mathrm{Y}$ for $\mathrm{Ca}$ decreases the hole concentration from the optimum value. Varoy et. al. have found a systematic fall to more negative values of thermoelectric power as holes are added with the introduction of more lead or oxygen into BISCCO compounds. In this chapter, we report the structural, electrical, magnetic and thermal transport properties of vanadium-substituted BISCCO (2223) systems.

\section{Experimental details}

In the Bi-based high- $\mathrm{T}_{\mathrm{c}}$ superconductors the Bi-2223 phase is stable within a narrow temperature range and exhibits phase equilibria with only a few of the compounds existing in the system. Precise control over the processing parameters is required to obtain the phasepure material (Rehman \& Maqsood, 2005). Bismuth-based superconducting powder with chemical formula $\mathrm{Bi}_{1.3} \mathrm{~V}_{0.3} \mathrm{~Pb}_{0.4} \mathrm{Sr}_{2} \mathrm{Ca}_{2} \mathrm{Cu}_{3} \mathrm{O}_{\delta}$ was prepared by the solid state reaction method. The starting powders corresponding to stoichiometric quantities of high purity $(99.9 \%)$ $\mathrm{Bi}_{2} \mathrm{O}_{3}, \mathrm{PbO}, \mathrm{SrCO}_{3}, \mathrm{CaCO}_{3}, \mathrm{~V}_{2} \mathrm{O}_{5}, \mathrm{CuO}$ were weighed on digital balance within $1 \mathrm{mg}$ accuracy. The powders were mixed and ground in an agate mortar and pestle for $2 \mathrm{~h}$ and then the mixed powders were calcined in air at $810^{\circ} \mathrm{C}$ for $24 \mathrm{~h}$. The porcelain crucibles were used for calcinations and sintering. The porcelain crucibles have a melting point of $1400^{\circ} \mathrm{C}$, are not contaminated with the materials being used and are economical. The aim of the calcination was to eliminate the carbonates and to produce an oxide with a nominal composition $\mathrm{Bi}_{1.3} \mathrm{~V}_{0.3} \mathrm{~Pb}_{0.4} \mathrm{Sr}_{2} \mathrm{Ca}_{2} \mathrm{Cu}_{3} \mathrm{O}_{\delta}$. The polyvinyl alcohol (PVA) was used as a binder for pellet formation. A pressure of $50 \mathrm{kN}$ was applied to all the pellets by a hydraulic press. The pellets with nominal composition $\mathrm{Bi}_{1.3} \mathrm{~V}_{0.3} \mathrm{~Pb}_{0.4} \mathrm{Sr}_{2} \mathrm{Ca}_{2} \mathrm{Cu}_{3} \mathrm{O}_{\delta}$ were finally sintered in air at 840 $\pm 5^{\circ} \mathrm{C}$ for $216 \mathrm{~h}$ in three steps (first step $96 \mathrm{~h}$, second step $72 \mathrm{~h}$ and the third $48 \mathrm{~h}$ ) and gradually cooled down to room temperature. Phase purity and lattice parameters were determined by X-ray diffraction (XRD). XRD data were taken using CuKa (1.5406 ̊) radiation. Grain size was analyzed from scanning electron microscopic (SEM) image taken on the surface on several cross-sectional cuts. No appreciable variations of size and aspect ratio of grains were observed for the different zones. DC electrical resistivity was used to characterize superconducting properties of the samples using standard four-probe DC 
technique, in the temperature range from 77 to $300 \mathrm{~K}$. The critical current density of the sample was measured by the four-probe method in such a way that the current may pass through the bulk of the specimen. For the calculation of $J c$ the following equation is used

$$
J_{c}=I_{c} / A=I_{c} /(W T)
$$

where $A$ is the area of cross section, $W$ is the width, $I_{c}$ is the critical current and $T$ is the thickness of the sample. Considerable care was taken to ensure that results were not affected by heating at the current contacts. The mutual inductance bridge method was used for AC magnetic susceptibility measurements. For these measurements, a very low AC field (0.5 Oe) was applied parallel to the axis of the rectangular bar-shaped specimen. The temperature range was again $77-300 \mathrm{~K}$. The thermal conductivity as a function of temperature was measured using the advantageous transient plane source techniques (ATPS). The detail of the ATPS technique is described in (Rehman \& Maqsood, 2005; Gusstafsson, 1991; Rehman, 2009) at length. Two identical sizes of the sample (diameter 25 $\mathrm{mm} \times$ thickness $10 \mathrm{~mm}$ ) were used for measurements. Thermoelectric power $\mathrm{S}(\mathrm{T})$ was obtained by taking the ratio of the voltage difference to the temperature gradient as

$$
S=\Delta V / \Delta T
$$

where $\Delta V$ is the voltage arising (in the absence of an applied field) across the sample with a temperature difference of $\Delta T$ between its ends, given by the relation

$$
S=E / \Delta T
$$

where $E$ is the electric field in the sample. The detail of the apparatus used is already published (Rehman \& Maqsood, 2005).

\section{Results and discussion}

X-ray diffraction (XRD) pattern of the sample with nominal composition $\mathrm{Bi}_{1.3} \mathrm{~V}_{0.3} \mathrm{~Pb}_{0.4} \mathrm{Sr}_{2} \mathrm{Ca}_{2} \mathrm{Cu}_{3} \mathrm{O}_{\delta}$ after the final sintering of $216 \mathrm{~h}$ is shown in Figure 2. XRD pattern was taken using $\mathrm{CuK} a$ radiation $(\lambda=1.5046 \AA)$ and measurements were taken at room temperature. All the peaks in the XRD pattern are indexed. XRD pattern showed peaks belonging to the high- $\mathrm{T}_{\mathrm{c}}$ (2223) phase as a major component along with peaks due to the low- $\mathrm{T}_{\mathrm{c}}$ (2212) phase. The dominant is the high- $\mathrm{T}_{\mathrm{c}}$ (2223) phase having orthorhombic structure with lattice constants: $a=5.417(6) \AA, b=5.392(7) \AA$ and $c=37.164(3) \AA$. The SEM result of the sample is shown in Figure 3.

It is clear from the figure that the superconducting grains are connected with each other, but with the unfilled spaces between them. This type of granular morphology has been rarely discovered in conventional high temperature crystalline superconducting samples. Furthermore, the average grain size was calculated at different spots of the sample and found to lie between 3 and $4 \mu \mathrm{m}$. The electrical property of the sample was examined by DC electrical resistivity measurements. The DC electrical resistivity as a function of temperature is also shown in Figure 4, after the final sintering step of $216 \mathrm{~h}$. The DC electrical resistivity measurements show a well defined metallic behavior and the superconducting transitions. The resistivity versus temperature plot shows that the resistivity decreases linearly with temperature in the normal state. The onset temperature $\mathrm{T}_{\mathrm{c}}$ (onset) and zero resistivity critical temperature $T_{c}(R=0)$ were found to be $112 \pm 1 \mathrm{~K}$ and $106 \pm 1 \mathrm{~K}$, respectively. The measured 


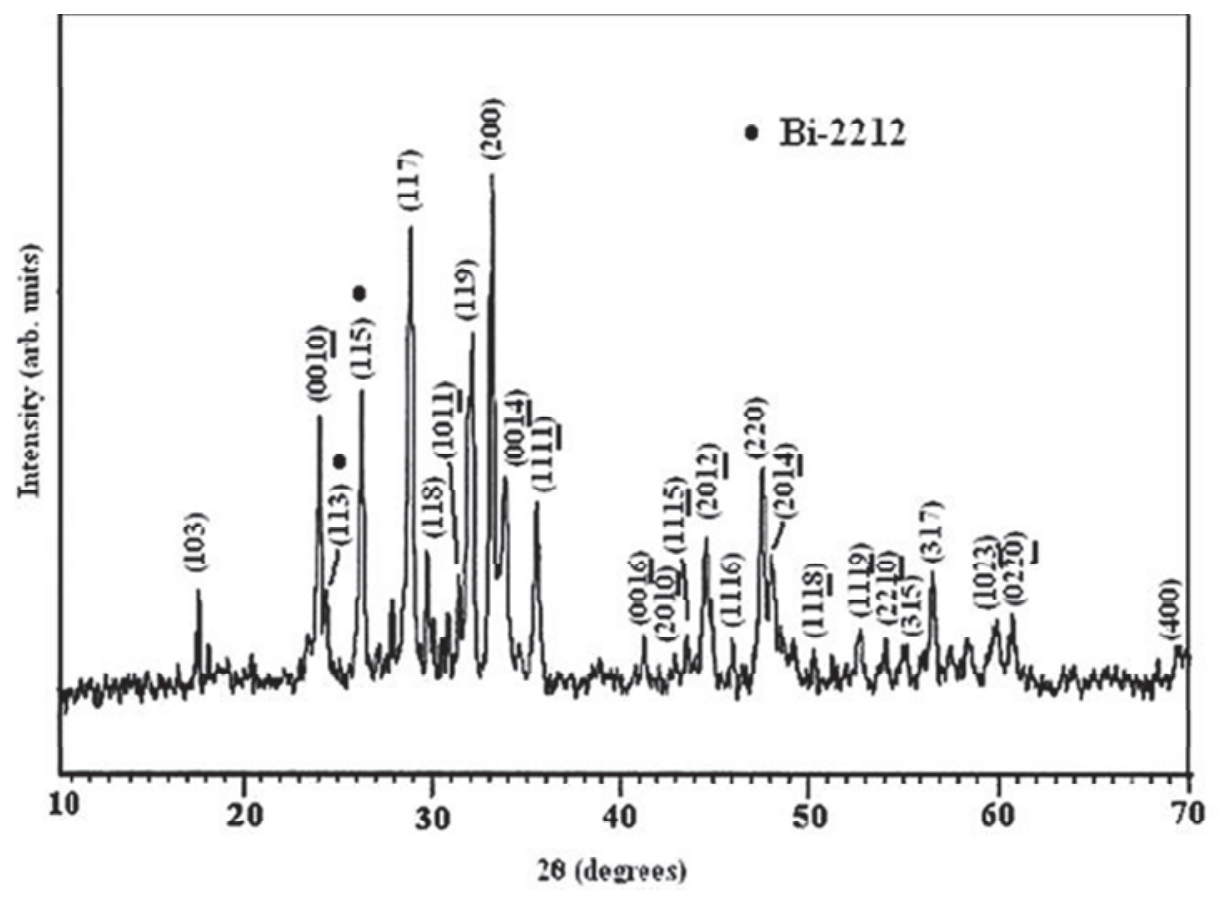

Fig. 2. Indexed X-ray pattern of $\mathrm{Bi}_{1.3} \mathrm{~V}_{0.3} \mathrm{~Pb}_{0.4} \mathrm{Sr}_{2} \mathrm{Ca}_{2} \mathrm{Cu}_{3} \mathrm{O}_{\delta}$ superconductor

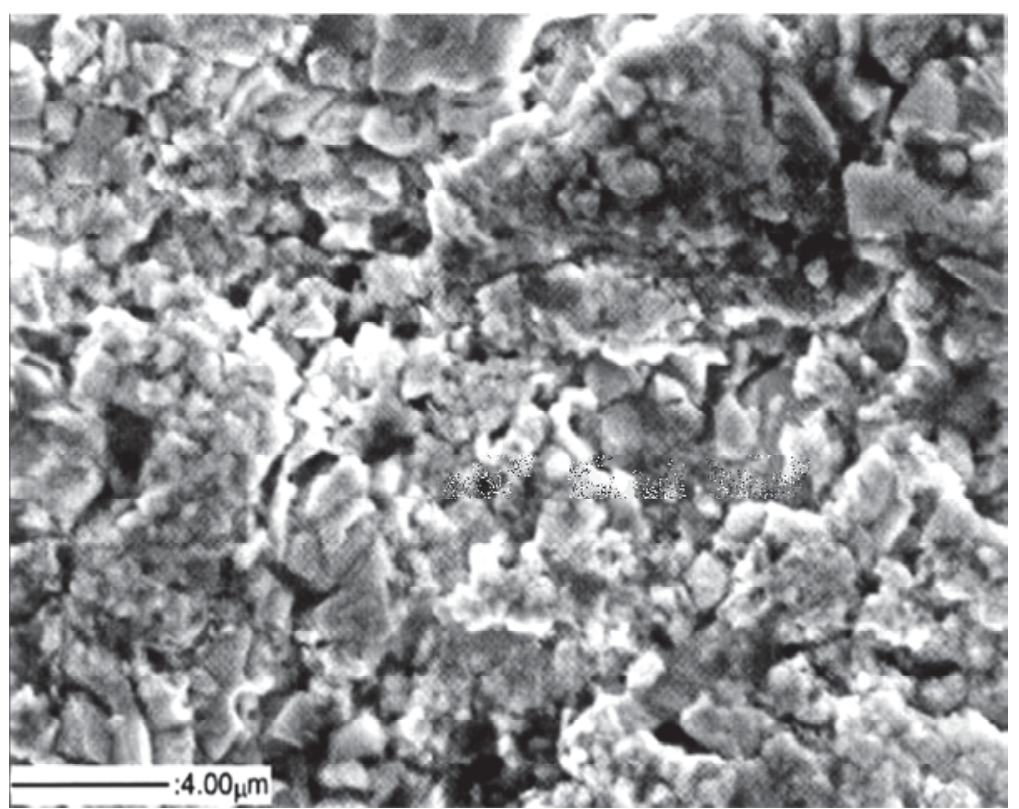

Fig. 3. SEM micrograph of the sample with composition $\mathrm{Bi}_{1.3} \mathrm{~V}_{0.3} \mathrm{~Pb}_{0.4} \mathrm{Sr}_{2} \mathrm{Ca}_{2} \mathrm{Cu}_{3} \mathrm{O}_{\delta}$ 


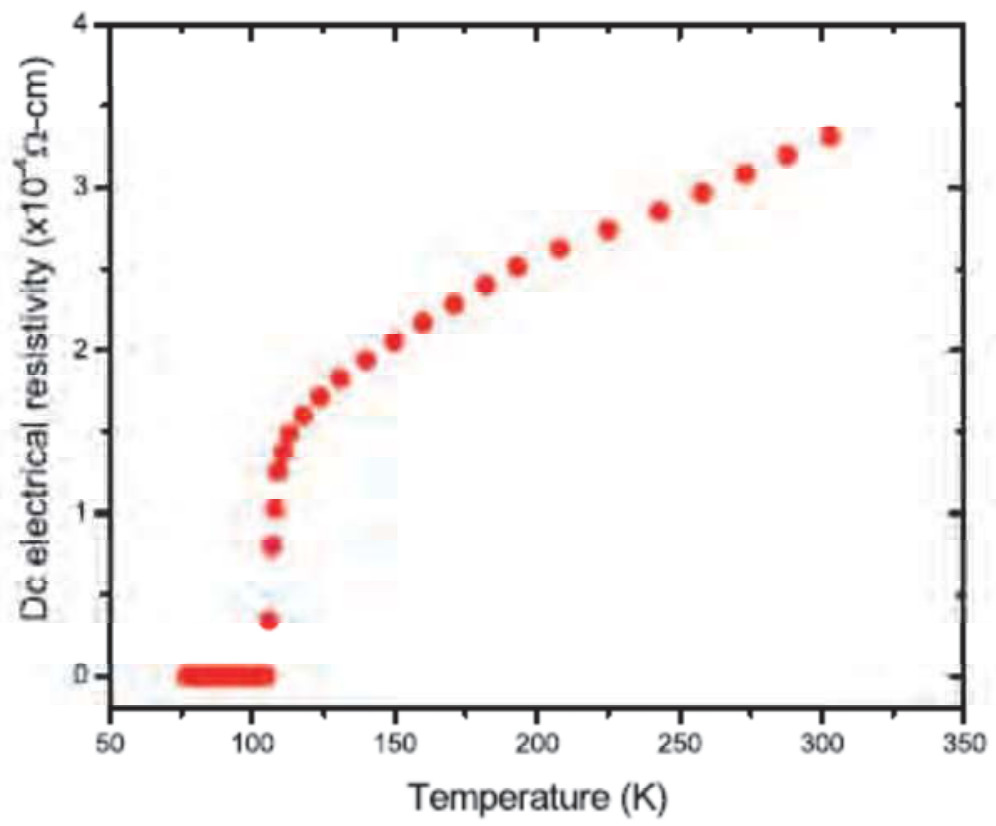

Fig. 4. Temperature dependence of DC electrical resistivity of the sample with nominal composition $\mathrm{Bi}_{1.3} \mathrm{~V}_{0.3} \mathrm{~Pb}_{0.4} \mathrm{Sr}_{2} \mathrm{Ca}_{2} \mathrm{Cu}_{3} \mathrm{O}_{\delta}$ after final sintering time of $216 \mathrm{~h}$

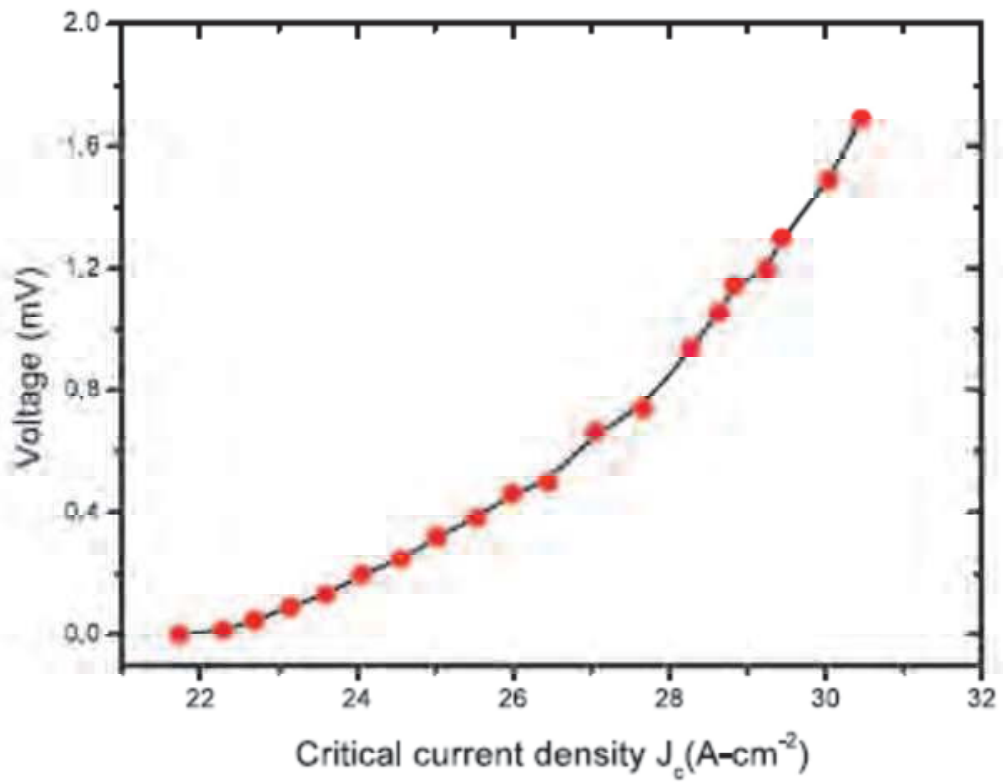

Fig. 5. Critical current density of the sample with composition $\mathrm{Bi}_{1.3} \mathrm{~V}_{0.3} \mathrm{~Pb}_{0.4} \mathrm{Sr}_{2} \mathrm{Ca}_{2} \mathrm{Cu}_{3} \mathrm{O}_{\delta}$ at $77 \mathrm{~K}$ 
mass density of the sample was $3.58 \pm 0.01 \mathrm{~g} / \mathrm{cm}^{3}$. The critical current density of the samples was measured at a temperature of $77 \mathrm{~K}$ in zero magnetic field as shown in Figure 5. It is clear that the value of the critical current density increases as the dopant concentration of vanadium increases, as compared to the undoped samples (Gul et. al., 2005). The increase in the critical current density is due to the fact that the increase in the concentration of vanadium increases the flux pinning, and strong coupling between the grain results in high values of critical current density $\left(J_{c}\right)$.

The measurement of the real part $\chi /$ of the $A C$ magnetic susceptibility on the sample $\mathrm{Bi}_{1.3} \mathrm{~V}_{0.3} \mathrm{~Pb}_{0.4} \mathrm{Sr}_{2} \mathrm{Ca}_{2} \mathrm{Cu}_{3} \mathrm{O}_{\delta}$ is clearly showing that there are two phases. The curve of Figure 6 displays a two-step process which reflects the flux penetration between and into the grains, as temperature decreases.

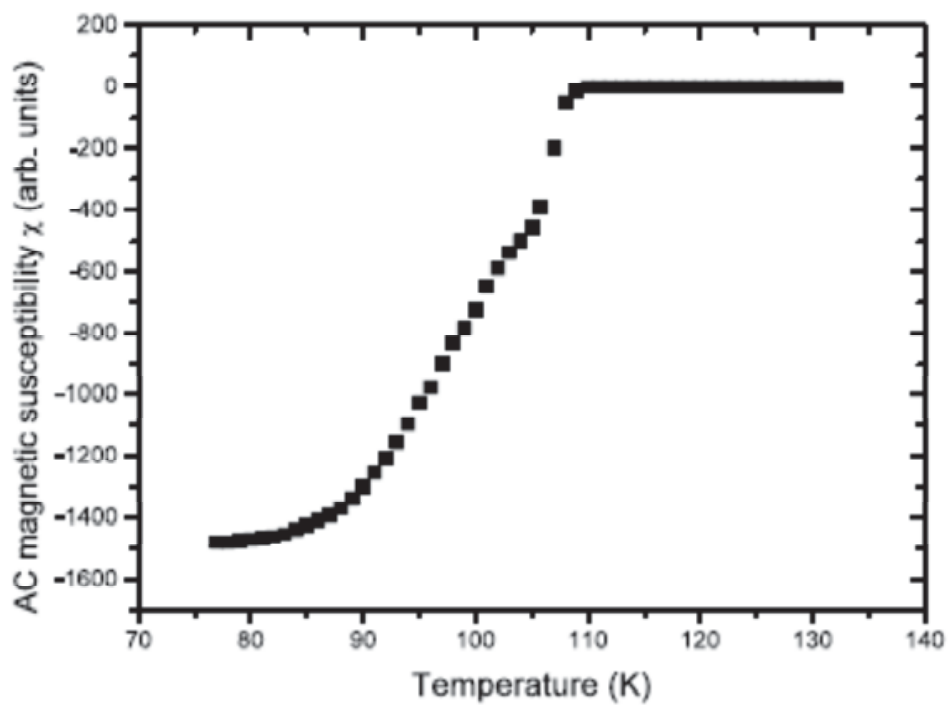

Fig. 6. Temperature dependence of the real part of AC susceptibility of the sample with composition $\mathrm{Bi}_{1.3} \mathrm{~V}_{0.3} \mathrm{~Pb}_{0.4} \mathrm{Sr}_{2} \mathrm{Ca}_{2} \mathrm{Cu}_{3} \mathrm{O}_{\delta}$

The diamagnetic transition in $\chi$ / seems to occur in the first phase with onset temperature $T_{c}$ (onset) around $113 \pm 1 \mathrm{~K}$. Another drop in diamagnetic signal is observed around $106 \pm 1 \mathrm{~K}$. This second phase has comparatively slow transition and it reflects the presence of low-Tc (2212) component, which is also confirmed by X-ray analysis. It is well known that the demagnetizing correction would cause $\chi^{\prime}=-1$ for a sufficiently low temperature. It is evident from Figure 2 that X-ray diffraction measurement supports the observation of AC magnetic susceptibility. When the sample is at a temperature just below $T_{c}(R=0)$, the superconducting grains shield the applied magnetic field. As the temperature decreases further, the inter-granular component $\chi /$ appears and rapidly grows proportionally to the quality of interconnectivity of the grains, and volume fraction of the sample is expected to be shielded by the super current circulates in the samples, and hence the curve of $\chi^{\prime}$ versus temperature saturates. Thermal conductivity measurement as a function of temperature ranging from 77 to $300 \mathrm{~K}$ is done using advantageous transient plane source (ATPS) technique. Thermal conductivity results are shown in Figure 7. 


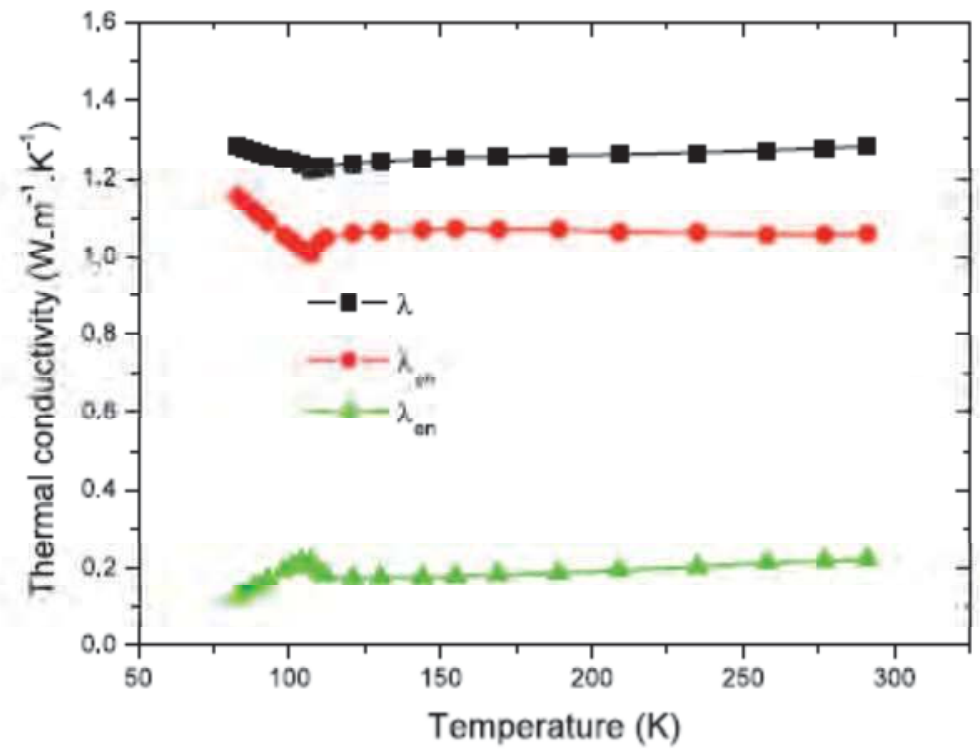

Fig. 7. Thermal conductivity $(\lambda)$ as a function of temperature along with electron $\left(\lambda_{\ell}\right)$ and phonon $\left(\lambda_{\text {ph }}\right)$ contributions

This plot shows that there is a change in thermal conductivity at $105 \pm 1 \mathrm{~K}$ corresponding to $T_{c}(R=0)$. There is an increase in slope close to $T_{c}(R=0)$, which is in good agreement with the literature (Aliev, et.al. 1992; Dey, et.al. 1991; Jezowski, 1992) and also agrees with the DC electrical resistivity and AC magnetic susceptibility measurements. In superconducting state the electrons, which become Cooper pairs, no longer are allowed to exchange energy with phonon; therefore, the lattice conduction rises as more and more Cooper pairs are formed. To find the electron-phonon scattering time $\left(\tau_{e-p h}\right)$, the relation reported by Hook and Hall (Hook \& Hall, 1991) is used as

$$
1 / \rho_{e-p h}=n e^{2} \tau_{e-p h} / m_{e}
$$

where $n, e$ and $m_{e}$ are the number density, charge and mass of the electron, respectively, $\rho_{e-p h}$ being the resistivity at a particular temperature. This gave $\tau_{e-p h}=2 \times 10^{-15} \mathrm{~s}$ at $118 \mathrm{~K}$, the temperature above which the resistivity is linear with temperature. Also, the phononlimited mobility can be calculated using the relation (Hook \& Hall, 1991)

$$
\mu_{e-p h}=e \tau_{e-p h} / m_{e}
$$

The calculated value of the phonon-limited mobility is $3 \times 10^{-4} \mathrm{C}-\mathrm{S}-\mathrm{Kg}^{-1}$. To estimate the size of the electron-phonon coupling constant, the lowest order variational solution of the Bloch-Boltzmann transport equation (Pinksi, et.al. 1981) is

$$
\hbar / \tau_{e-p h}=2 \pi d_{e-p h} k_{B} T
$$

here $d e-p h$ is the electron-phonon coupling constant which is 6. These results are in good agreement with the results reported by other authors (Heremans, et.al. 1988; Suleiman, et.al. 1993). The heat flux in solids consists of two contributions: energy transport associated with 
the flow of the charge carriers that are referred to as the electronic thermal conductivity, and thermal energy carried by lattice vibrations that is known as the lattice or phonon thermal conductivity. As is well known, the thermal conductivity $(\lambda)$ of metals is given by these two contributions:

$$
\lambda=\lambda_{e}+\lambda_{\text {ph }}
$$

here $\lambda_{e}$ is the thermal conductivity due to electrons and $\lambda_{p h}$ is the thermal conductivity due to phonons. In simple metals, the separation of the two components of the thermal conductivity is made by use of the Wiedemann-Franz (WF) law (Ziman, 1963),

$$
\lambda_{\text {en }} \rho / T=(1 / 3) \pi^{2}\left(k^{2} B / e^{2}\right)=2.45 \times 10^{-8} \text { W. } . \mathrm{K}^{-2}
$$

where, $\lambda_{e n}$ is the electronic thermal conductivity in the normal state, $\rho$ is the electrical resistivity, $k_{B}$ is the Boltzmann constant. In ordinary metals, the WF law fails at intermediate temperatures where the electrical resistivity $\rho$ deviates from the $T$ linear dependence. The electrical resistivity of oxide superconductors shows the characteristic $T$ linear dependence over quite a wide temperature range, as can be seen in Figure 4. Accordingly, the WF law is expected to hold over the entire temperature range above $T_{c}(R=0)$ for the oxide superconductors and to result in constant and small $\lambda_{\text {en }}$, making a marked contrast to ordinary metals. Below $T_{c}(R=0)$ the charge carriers that have condensed in the ground state do not contribute to the heat conduction, and the electronic component $\lambda_{e s}$ is expected to decrease with lowering temperature. Among several theories (Bardeen, et.al. 1962; Tewordt, 1963) that treat $\lambda_{e s}$, we refer to revised Kadanoff's formulation adapted by Ikebe et al. (Ikebe, et.al. 1994) to observe the linear dependence of $\rho$,

$$
\lambda_{e s} / \lambda_{e n}=\left(3 / 2 \pi^{2}\right) \int_{0}^{\infty} d \varepsilon \varepsilon^{2} \sec h^{2}\left\{1 / 2\left[\varepsilon^{2}+(\beta \Delta)^{2}\right]^{1 / 2}\right\} \times\left[1+\mathrm{a}\left(\mathrm{T} / \mathrm{T}_{\mathrm{c}}\right) /\left(\varepsilon /\left[\varepsilon^{2}+(\beta \Delta)^{2}\right)\right]^{1 / 2}+\mathrm{a}\left(\mathrm{T} / \mathrm{T}_{\mathrm{c}}\right)\right]
$$

here, $a$ represents the ratio of the $T$-linear electrical resistance at $T_{C}(R=0)$ to the residual resistance, $\beta=1 / k_{B} T$, and $\Delta$ is the BCS energy gap in the Buckingham (Bardeen, et.al. 1957; Buckingham, 1956) form,

$$
\Delta=3.2 k_{B} T_{c}\left(1-T / T_{c}\right)^{1 / 2}
$$

The electronic and phonon contribution to thermal conductivity is estimated by (7), (8) in normal and superconducting state, respectively, as is shown in Figure 7, along with $\lambda$. The thermoelectric power is used as a medium to investigate the physical properties, in particular the properties that can be correlated with the transport mechanism that occurs in these superconductors. Thermoelectric power is a sensitive property in high-temperature superconductors, particularly to determine the sign of the charge carriers. The apparatus for the measurement of thermoelectric power was being assembled. In the aim to test the apparatus, it was calibrated with copper. Figure 8 shows that the behavior of the thermoelectric power of copper is almost linear with temperature. The magnitude of thermoelectric power of copper is approximately what one expects in noble metals such as silver and gold.

The thermoelectric power of these metals is positive except at very low temperatures. The positive sign of these metals is due to that of the Fermi surfaces touching the Brillouin zone boundaries. The Fermi surface areas could decrease with an increase of the electron energy (Barnard, 1972). Thermoelectric power (thermopower or Seebeck coefficient) determines the interaction between electrical and thermal currents in a conductor. The phonon-phonon 


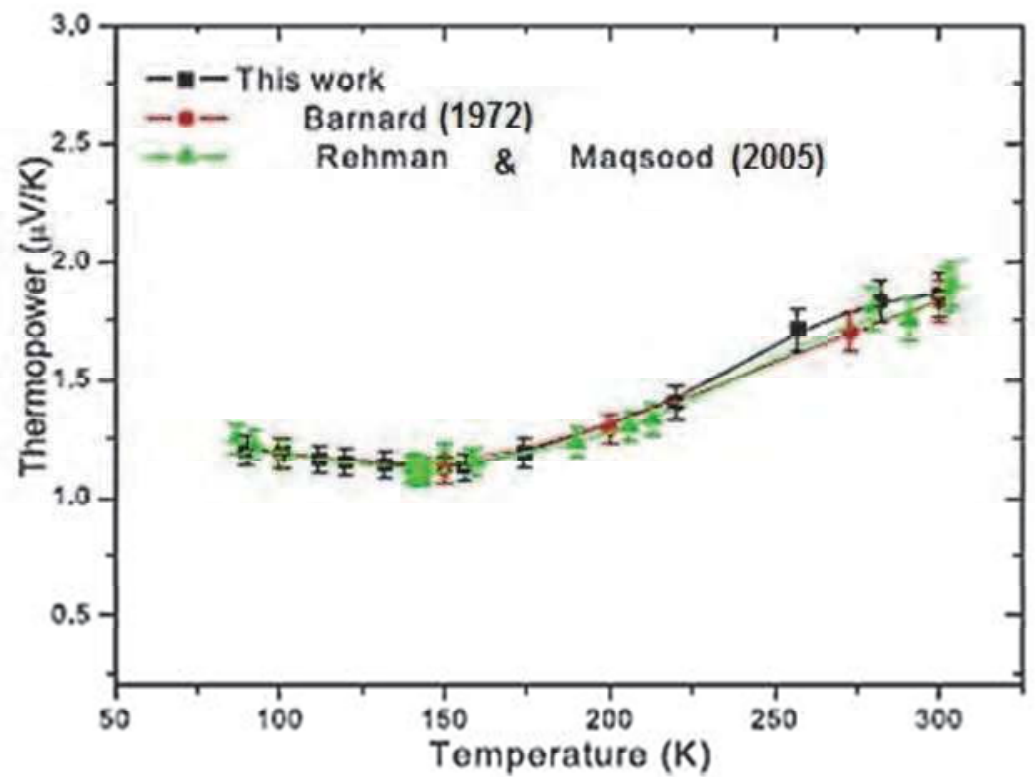

Fig. 8. Absolute thermoelectric power of copper as a function of temperature

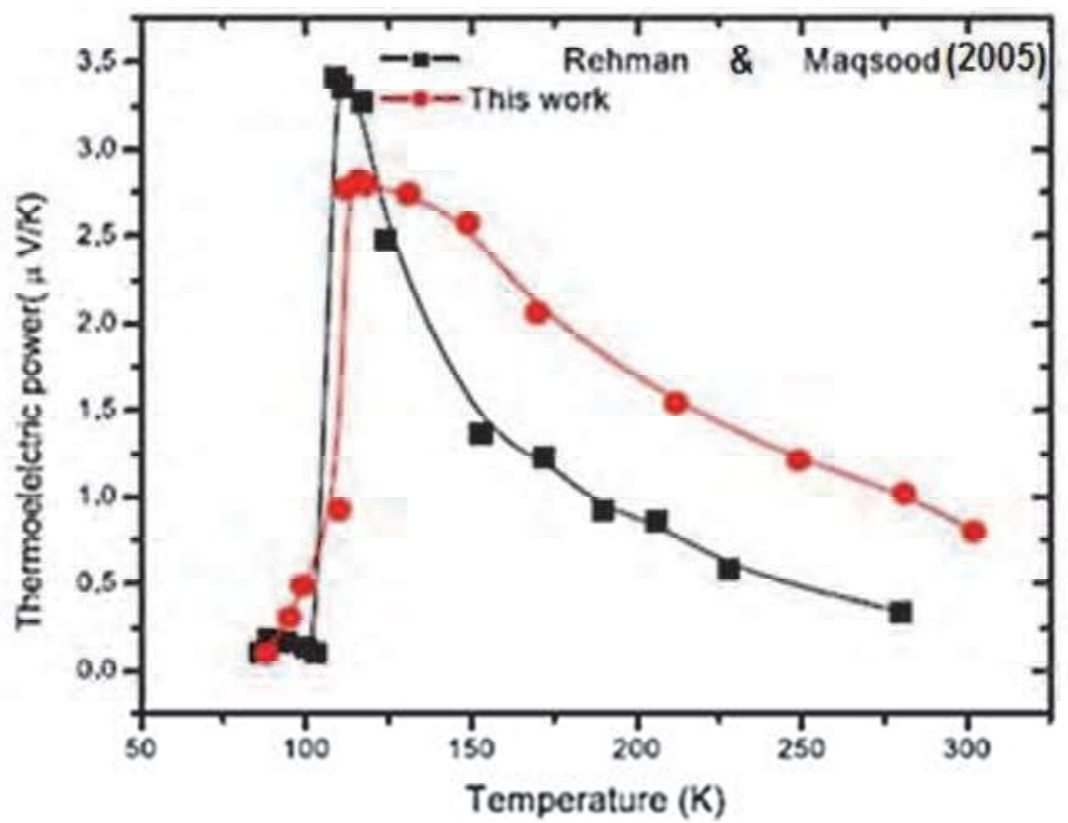

Fig. 9. Thermoelectric power of the sample with composition $\mathrm{Bi}_{1.3} \mathrm{~Pb}_{0.4} \mathrm{~V}_{0.3} \mathrm{Sr}_{2} \mathrm{Ca}_{2} \mathrm{Cu}_{3} \mathrm{O}_{\delta}$ 
interaction is dominant at higher temperature, arises from anharmonicity in potential and this increase with temperature. At low temperature phonon-phonon interaction is less important. The precise form of the curve at very low temperatures is uncertain because the thermopower increases rapidly associated with the metal impurities, notably iron. Thus minute quantities of iron were the cause of this increase in thermopower even in the ultrapure copper at very low temperature (Gul, et. al., 2005). Thermoelectric power of the superconducting sample measured in the temperature range $85-300 \mathrm{~K}$ is shown in Figure 9.

From the figure, it is clear that thermoelectric power increases with decreasing temperature and drops rapidly to zero at superconducting transition phase. From transition to room temperature, thermoelectric power is closely linear with temperature and its profile is similar to that of other high-Tc superconductors (Mitra \& Trefny, 1988). The critical temperature in thermoelectric power is observed at $116 \pm 1 \mathrm{~K}$, after that thermoelectric power begins to decrease and becomes almost zero at $88 \pm 1 \mathrm{~K}$. The critical temperature $T_{c}$ already determined from electrical resistivity was $106 \pm 1 \mathrm{~K}$. To explain these results, we assume that two kinds of carriers existed simultaneously in our sample: electrons and holes. The values of thermoelectric power in our sample are very small so we can say that a very week Seebeck coefficient shows that the behavior of the sample is typically metallic. In any superconductor, two types of contributions are important: one from phonons and the other from electrons. But contribution due to phonons is not important above the liquid nitrogen temperature (Yan, et.al., 1988).

It is also noted that the onset temperature drops of thermoelectric power are higher than those of the resistance drops. A possible explanation might be as follows. Since these materials are granular, one expects high electrical resistance between grains. On the other hand, the temperature drops between grains are expected to be small and, consequently, the granular nature would have less effect on thermoelectric power than the electrical resistivity (Lim, et,al. 1989). Polycrystalline HTSCS are generally viewed as an agglomeration of grains compacted together. On deoxygenation, the surface of the grains loses more and more oxygen than the grain itself. For $\mathrm{T}<\mathrm{T}_{\mathrm{c}}(\mathrm{R}=0)$, the grains become good superconductors where they are coupled together by weak links formed within the intergranular region. The weak links behave as superconducting region with very small critical current. These links become weaker and weaker with increasing deoxygenation. In such a situation, a very small current used in the critical resistivity measurement can exceed the $J c$ of the weak links, resulting in the breakdown of the percolation chain. Consequently, the resistance can persist. The temperature $T_{c}(R=0) S$ is lower than the $T_{c}(R=0)$ of the bulk material as long as $J c$ of the weak links is smaller than the transport current density. However, contrary to the resistivity measurement, the thermopower experiment is performed in the absence of any current. Hence the percolation chain of the superconducting grains can persist up to temperatures higher than $T_{c}(R=0)$ RES even in the presence of a small temperature gradient. This shifts $T_{c}(R=0) S$ to relatively higher temperatures (Dey, et. al., 1998).

\section{Conclusions}

A superconducting sample with nominal composition $\mathrm{Bi}_{1.3} \mathrm{~V}_{0.3} \mathrm{~Pb}_{0.4} \mathrm{Sr}_{2} \mathrm{Ca}_{2} \mathrm{Cu}_{3} \mathrm{O}_{\delta}$ was prepared by the solid state reaction method. By resistivity measurement, $T_{c}(R=0) R E S$ obtained for this composition was $106 \pm 1 \mathrm{~K}$. Thermal conductivity variation with temperature showed initially a slight decrease and then a pronounced increase around $T_{c}(R$ $=0)$. Although the expected theoretical trend is similar, the peak near T/2 was not observed 
due to temperature limitations of the temperature controller. A similar behavior is observed in all hole-type $\mathrm{CuO}_{2}$ plane superconductors and in all their structural forms. This effect is due to phonon or quasiparticle scattering. The Wiedemann-Franz law is applied to predict the magnitude of electron and phonon contributions to the total thermal conductivity of the samples, whereas $T_{c}$ (onset) observed was at $112 \pm 1 \mathrm{~K}$. Thermoelectric power of the sample was positive in the temperature range $85-300 \mathrm{~K}$. The behavior of thermoelectric power in this superconducting sample was approximately linear with temperature as observed in other Bismuth-based high- $\mathrm{T}_{\mathrm{c}}$ superconductors. The transition temperature of this superconductor $T_{c}(R=0) S$ was measured to be $116 \pm 1 \mathrm{~K}$, whereas the transition temperature of this sample measured with resistivity, $T_{c}(R=0) R E S$, method was $106 \pm 1 \mathrm{~K}$. The difference between $T_{c}(R=0) R E S$ and $T_{c}(R=0) S$ is $10 \mathrm{~K}$. This difference increases as more and more oxygen is being taken out of the compound by deoxygenation.

\section{Acknowledgements}

The authors would like to acknowledge the Higher Education Commission (HEC) Islamabad, Pakistan for the financial support. Mr. A. Abdullah is acknowledged for useful support in preparation of the manuscript.

\section{References}

Aksan, M.A., Yakinci, M.E., Balci, Y. \& Ates, H. (1999). Thermal conductivity properties of $\mathrm{Bi}_{2-\alpha} \mathrm{Tl}_{\alpha} \mathrm{Sr}_{2} \mathrm{Ca}_{2} \mathrm{Cu}_{3} \mathrm{O}_{10+z}$ glass-ceramic $\mathrm{HT}_{\mathrm{c}}$ superconductor rods. J. Low Temp. Phys. Vol. 117, No. 3-4, 957-961

Aksan, M.A. \& Yakyncy, M.E. (2004). Synthesis and characterization of Er-substituted Bi-2223 H-T $T_{\text {c }}$ glass-ceramic superconductors. J. Alloy. Compd. Vol. 385, No. 1-2, 33-43

Aliev, F.G., Moshchalkov, V.V., Pryadun, V.V. \& Monge, M.A.A. (1992). Thermal conductivity of $\mathrm{TmBa}_{2} \mathrm{Cu}_{3} \mathrm{O}_{x}$ and $\mathrm{Bi}_{2} \mathrm{Sr}_{2} \mathrm{CaCu}_{2} \mathrm{O}_{x}$ single crystals in the vicinity of the superconducting transition. Solid State Commun. Vol. 82, No. 4, 241-244

Bardeen, J., Cooper, L.N. \& Schrieffer, J.R. (1957). Theory of superconductivity Phys. Rev. Vol. No. 5, 108, 1175-1204

Bardeen, J., Cooper, L.N., Schrieffer, J.R. (1962). Theory of the thermal conductivity of superconductors. Phys. Rev. Vol. 113, No. 4, 982-994

Barnard, R.D.: Thermoelectricity in Metals and Alloys. Taylor and Francis Ltd., London (1972)

Buckingham, M.J. (1956). Very high frequency absorption in superconductors Phys. Rev. Vol. 101, No. 4, 1431-1432

Chanda, B. \& Dey, T.K. (1994). Thermal conductivity of bismuth-lead (2223) superconducting cuprates with vanadium/nickel substitutions and evidence for strong electron-phonon coupling. Solid State Commun. Vol. 89, No. 4, 353-361

Castellazzi, S., Cimberle, M.R., Ferdeghini, C., Giannini, E., Grasso, G., Marre, D., Putti, M. \& Siri, A.S. (1997). Thermal conductivity of a $\operatorname{BSCCO}(2223) c$-oriented tape: a discussion on the origin of the peak. Physica C Vol. 273, No. 3-4, 314-322

Chatterjee, S., Pal, P.K., Bhattacharya, S. \& Chaudhuri, B.K. (1998). Depression of superconductivity and the phonon-drag effect in glass-ceramic superconductors 
obtained by annealing the Fe-doped $(\mathrm{Bi}, \mathrm{Pb})_{4} \mathrm{Sr}_{3} \mathrm{Ca}_{3} \mathrm{Cu}_{4-m} \mathrm{Fe}_{m} \mathrm{O}_{x}$ glassy precursor. Phys. Rev. B. Vol. 58, No. 18, 12427-12432

Cloots, R., Bougrine, H., Houssa,M., Stassen, S., D’Urzo, L., Rulmont, A. \& Ausloss, M. (1994). Bi-based 2223 superconducting polycrystalline materials prepared by either a solid state route or a glassy 'matrix' precursor method: Chemical analysis as well as electrical and thermal transport properties. Physica C. Vol. 231, No. 34, 259-270

Cohn, J.L., Skelton, E.F., Wolf, S.A., Liu, J.Z. \& Shelton, R.N. (1992). Cyclotron resonance of both magnetopolaron branches for polar and neutral optical phonon coupling in the layer compound InSe.Phys. Rev. B vol. 45, No. 20, 12144-12147

Coskun, A., Ekicibil, A., Ozcelik, B. \& Kyymaç, K. (2005). Effects of annealing time on the magnetic properties of a $\mathrm{Bi}_{1.7} \mathrm{~Pb}_{0.29} \mathrm{Gd}_{0.01} \mathrm{Sr}_{2} \mathrm{Ca}_{3} \mathrm{Cu}_{4} \mathrm{O}_{12+}$ y superconductor prepared by the melt-quenching method. Chin. J. Phys. Vol. 43, No. 2, 372

Dey, T.K. (1998). Thermoelectric power of deoxygenated $\mathrm{Bi}_{1.6} \mathrm{~Pb}_{0.4} \mathrm{Sr}_{2} \mathrm{Ca}_{2} \mathrm{Cu}_{3} \mathrm{O}_{10+\delta}$ sintered superconducting pellets. J. Supercond. Vol.11, 367-372

Dorbolo, S., Ausloss, M., Bougrine, H., Robertz, B., Cloots, R., Mucha, J. \& Durczewski, K. (1999). Effect of synthesis process and substrate on electrical and thermal transport properties of Bi-2212. J. Supercond. Vol. 12, Number 5, 623-629

Ekicibil, A., Coskun, A., Ozcelik, B. \& Kyymacç, K. (2005). The magnetic and electrical properties of rare earth $\mathrm{Sm}^{3+}$ substituted $\mathrm{Bi}_{1.7} \mathrm{~Pb}_{0.3} \mathrm{Sr}_{2} \mathrm{Ca}_{2-x} \mathrm{Sm}_{x} \mathrm{Cu}_{3} \mathrm{O}_{12}$ system. Mod. Phys. Lett. B Vol. 331, No. 6, 331-340

Gul, I.H., Rehman, M.A., Ali, M. \& Maqsood, A. (2005). Effect of vanadium and barium on the Bi-based (2223) superconductors. Physica C Vol. 432, No. 1-2, 71-80

Gusstafsson, S.E.(1991). Transient plane source techniques for thermal conductivity and thermal diffusivity measurements of solid materials. Rev. Sci. Instrum. Vol. 62, No. 3, 797-804

Heremans, J., Morelli, D.T., Smith, G.W., Strite, S.C. (1988). Thermal and electronic properties of rare-earth $\mathrm{Ba}_{2} \mathrm{Cu}_{3} \mathrm{O}_{x}$ superconductors. Phys. Rev. B Vol. 37, 16041610

Hirai, T.: In: Brook, R.J. (ed.) (1996). Processing of Ceramics, Part 2. VCH Verlagsgesellschaft $\mathrm{mbH}$, Weinheim

Hook, J.R., Hall, H.E. (1991). Solid State Phys, 2nd edn. Wiley, Chichester

Houssa, M. \& Ausloos, M. (1995). Thermal conductivity of superconducting $\mathrm{Bi}_{2} \mathrm{Sr}_{2} \mathrm{CaCu}_{2} \mathrm{O}_{8}$ and $\mathrm{YBa}_{2} \mathrm{Cu}_{3} \mathrm{O}_{7-y}$. Phys. Rev. B Vol. 51, No. 14, 9372-9374

Houssa, M. \& Ausloos, M. (1996). n-plane electronic thermal conductivity of layered d-wave high- $T_{\mathrm{c}}$ superconductors. Physica C Vol. 257, No. 3-4, 321-331

Houssa,M., Ausloos,M. \& Sergeenkov, S. (1996). The electronic contribution to the thermal conductivity of layered high- $\underline{T}_{\underline{c}}$ materials. J. Phys.: Condens. Phys. Vol. 8, No. 12, 2043-2052

Hui, P.M., Zhang, X. ,Markworth, J. \& Stroud, D. (1999). Thermal conductivity of graded composites: Numerical simulations and an effective medium approximation. $J$. Mater. Sci. Vol. 34, Number 22, 5497-5503 
Ikebe, M. Fujisshiro, H. Naito, T., Noto, K. (1994). Simultaneous measurement of thermal diffusivity and conductivity applied to Bi-2223ceramic superconductor. J. Phys. Soc. Jpn. Vol. 63, 3107-3114

Jezowski, A., Mucha, J., Rogaci, K., Horyn, R., Bukovski, Z. \& Horobiowski, M. (1987). Thermal conductivity and electrical resistivity of the high- $T_{c}$ superconductor $\mathrm{YBa}_{2} \mathrm{Cu}_{3} \mathrm{O}_{9-\Delta}$. Phys. Lett. A Vol. 122, No. 8, 431-433

Khim, Z.G., Lee, S.C., Lee, J.H., Suh, B.J., Park, Y.W., Park, C., Yu, I.S. \& Park, J.C. (1987). Superconductivity in single-phase $\mathrm{Y}_{1} \mathrm{Ba}_{2} \mathrm{Cu}_{3} \mathrm{O}_{9-x}$ and thermoelectric power measurement. Phys. Rev. B Vol. 36, No. 4, 2305-2307

Knizek, K., Veverka, M., Hadova, E., Hejtmanek, J., Sedmidubsky, D. \& Pollert, E. (1998). Synthesis of $\mathrm{HgBa}_{2} \mathrm{CuO}_{4+\delta}$ by sol-gel method under controlled oxygen pressure; electron and thermal transport properties. Physica C Vol. 302, No. 4, 290-298

Lee, S.C., Lee, J.H., Suh, B.J., Moon, S.H., Lim, C.J. \& Khim, Z.G. (1988). Thermoelectric

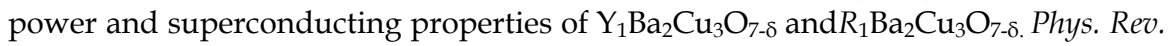
$B$ Vol. 37, No. 4, 2285-2288

Lim, Z.S., Han, K.H., Salk, S.H. \& Song, Y.S. (1989). Charged-particle interaction with liquids: ripplon excitations. Phys. Rev. B Vol. 40, No. 1, 10-19

Mandal, J.B., Keshri, S., Mandal, P., Poddar, A., Das, A.N. \& Ghosh, B. (1992). Thermoelectric power of the $\mathrm{Bi}_{2} \mathrm{Sr}_{2} \mathrm{Ca}_{1-x} \mathrm{Y}_{x} \mathrm{Cu}_{2} \mathrm{O}_{8+y}(x=0-1.0)$ system. Phys. Rev. B Vol. 46, No. 18, 11840-11846

Maqsood A., Mahmood M.S., Suleman B. \& Tasneem A. (1989). Preparation and characterization of superconducting $\mathrm{Dy}_{1} \mathrm{Ba}_{2} \mathrm{Cu}_{3} \mathrm{O}_{7-} x$ material. J. Mat. Sci. Lett. Vol. 8, No. 7, 757-758

Mitra, N. \& Trefny, J. (1988). Thermoelectric power of the Tl-Ca-Ba-Cu-O superconductor. Phys. Rev. B Vol. 38, No. 10, 7064-7066

Morelli, D.T., Heremans, J. \& Swets, D.E. (1987). Thermal conductivity of superconductive Y-Ba-Cu-O. Phys. Rev. B Vol. 36, No. 7, 3917-3919

Munakata, F., Matsuura, K., Kubo, K., Kawano, T. \& Yamauchi, H. (1992). Thermoelectric power of $\mathrm{Bi}_{2} \mathrm{Sr}_{2} \mathrm{Ca}_{1-x} \mathrm{Y}_{x} \mathrm{Cu}_{2} \mathrm{O}_{8+y}$. Phys. Rev. B Vol. 45, No. 18, 10604-10608

Nanda Kishore, K., Satyavathi, S., Muralidhar, A., Pena, O. \& Hari Babu, V. (1995). Thermoelectric power studies on the Sm substituted BPSCCO (2223) superconductors. Physica C Vol. 252, No. 1-2, 49-53

Natividad, E., Castro, M., Burriel, R., Angurel, L.A., Diez, J.C. \& Navarro, R. (2002). Correlation of normal and superconducting transport properties on textured Bi2212 ceramic thin rods. Supercond. Sci. Technol. Vol. 15, No. 7, 1022

Ozhanli, Z., Yakýncý, M.E., Balcý, Y. \& Aksan, M.A. (2002). Crystallization activation energy and hole concentration properties of the $\mathrm{Bi}_{2} \mathrm{Sr}_{2} \mathrm{Ca}_{1}-{ }_{\mathrm{x}} \mathrm{Cd}_{\mathrm{x}} \mathrm{Cu}_{2} \mathrm{O}_{8}+\mathrm{y}$ glassceramic superconductor Rods. J. Supercond. Vol. 15, No. 6, 543-547

Peacor, S.D., Cohn, J.L. \& Uher, C. (1991). Effect of magnetic field on thermal conductivity of $\mathrm{YBa}_{2} \mathrm{Cu}_{3} \mathrm{O}_{7-\delta}$ single crystals. Phys. Rev. B Vol. 43, No. 10, 8721-8724

Pinksi, F.J., Allen, P.B. \& Butler, W.H. (1981). Calculated electrical and thermal resistivities of $\mathrm{Nb}$ and Pd. Phys. Rev. B Vol. No. 10,23, 5080-5096 
Rao, C.N.R., Ramakrishnan, T.V. \& Kumar, N. (1990). Systematics in the thermopower behaviour of several series of bismuth and thallium cuprate superconductors: An interpretation of the temperature variation and the sign of the thermopower. Physica C Vol. 165, No. 2, 183-188

Rehman, M.A. \& Maqsood, A. (2005). Study of the thermal behaviour of $\mathrm{Bi}(\mathrm{Pb}) \mathrm{Sr}(\mathrm{Ba})-2223$ high- $T_{\mathrm{c}}$ granular superconductors. Physica C. Vol. 418, No. 3-4, 121-130

Rehman M.A. (2009). Thermal and electrothermal characterization of bismuth based high$T_{\mathrm{c}}$ superconductors J. Alloys Comp. Vol. 469, No. 1-2, 66-72

Sera,M., Tanaka, S., Sato,M. \& Fujishita, H. (1992). Anomalous thermoelectric power of $\mathrm{Bi}_{1.6} \mathrm{~Pb}_{0.5} \mathrm{Sr}_{1.9-\mathrm{y}} \mathrm{La}_{\mathrm{y}} \mathrm{Cu}_{1.05} \mathrm{O}_{z}$. Solid State Commun. Vol. 81, No. 5, 415-417

Suleiman, B.M., Haq, I., Karawacki, E.,Maqsood, A. \& Gustafsson, S.E. (1993). Thermal conductivity and electrical resistivity of the Y- and Er-substituted 1:2:3 superconducting compounds in the vicinity of the transition temperature. Phys. Rev. B Vol. 48, No. 6, 4095-4102

Tewordt, L. (1963). Theory of the intrinsic electronic thermal conductivity of superconductors Phys. Rev. Vol. 129, No.2, 657-663

Tewordt, L. \& Wolkhausen, T. (1989). Theory of thermal conductivity of the lattice for high$T_{c}$ superconductors. Solid State Commun. Vol. 70, No. 8, 839-844

Tewordt, L. \& Wolkhausen, T. (1990). Theory of phonon thermal conductivity for strongcoupling s- and d-wave pairing in high $\mathrm{T}_{\mathrm{c}}$ superconductors. Solid State Commun. Vol. 75, No. 6, 515-519

Uher, C. \& Kaiser, A.B. (1987). Thermal transport properties of $\mathrm{YBa}_{2} \mathrm{Cu}_{3} \mathrm{O}_{7}$ superconductors. Phys. Rev. B Vol. 36, No. 10, 5680

Uher, C. \& Wang, W.N. (1989). Thermoelectric power and thermal conductivity of neutronirradiated $\mathrm{YBa}_{2} \mathrm{Cu}_{3} \mathrm{O}_{7-\delta}$, Phys. Rev. B Vol. 40, No. 4, 2694-2697

Uher, C.: In: Ginsberg, D.M. (ed.) Physical Properties of HTSC,vol. 3. World Scientific, Singapore (1992)

Vasudeva R. V., Rangarajan, G. \& Srinivasan, R. (1984). Thermoelectric power in the normal state of Chevrel-phase superconductors of the type $\mathrm{Cu}_{1.8} \mathrm{Mo}_{6} \mathrm{~S}_{8-\mathrm{y}} \mathrm{Se}_{\mathrm{y}}, 0 \leq \mathrm{y} \leq 8$, and $\mathrm{Cu}_{1.8} \mathrm{Mo}_{6} \mathrm{~S}_{8-\mathrm{y}} \mathrm{Te}_{\mathrm{y}} 0 \leq \mathrm{y} \leq 4$. J Phys.F Vol.14, 973-980

Varoy, C.R., Trodahl, H.J., Buckley, R.G. \& Kaiser, A.B. (1992). Thermopower of $\mathrm{Bi}_{2-x} \mathrm{~Pb}_{x} \mathrm{Sr}_{2} \mathrm{CaCu}_{2} \mathrm{O}_{8+\delta}$. Phys. Rev. B Vol. 46, No. 1, 463-468

Yan, S., Lu, P. \& Li, Q. (1988). Thermoelectric power of single phase_ $\mathrm{YBa}_{2} \mathrm{Cu}_{3} \mathrm{O}_{7-x}$ superconductors. Solid State Commun. Vol. 65, No. 5, 355-358

$\mathrm{Yu}$, R.C., Salamon, M.B., Lu, J.P. \& Lee, W.C. (1992). Thermal conductivity of an untwinned $\mathrm{YBa}_{2} \mathrm{Cu}_{3} \mathrm{O}_{7-\delta}$ single crystal and a new interpretation of the superconducting state thermal transport. Phys. Rev. Lett. Vol. 69, No. 9, 14311434

Varoy, C.R., Trodahl, H.J., Buckley, R.G. \& Kaiser, A.B. (1992). Thermopower of Bi ${ }_{2-}$ ${ }_{x} \mathrm{~Pb}_{x} \mathrm{Sr}_{2} \mathrm{CaCu}_{2} \mathrm{O}_{8+\delta}$. Phys. Rev. B Vol. 46, No. 1, 463-468

Wang, J., Wakata, M., Kaneko, T., Takano, S. \& Ya-mauchi, H.(1993). Enhancement of $T_{\mathrm{c}}$ in $(\mathrm{Bi}, \mathrm{Pb})-2223$ superconductor by vacuum encapsulation and post-annealing. Physica C vol. 208, No. 3-4, 323-327 
Wermbter, S. \& Tewordt, L. (1991). Theory of thermal conductivity and nuclear relaxation rate for high temperature superconductors. Physica C Vol. 183, No. $4-6,365-371$

Wu M.K., Ashburn J.R., Torng C.J. \& Horetal P.H. (1987). Superconductivity at $93 \mathrm{~K}$ in a new mixed-phase Y-Ba-Cu-O compound system at ambient pressure. Phys. Rev. Lett. Vol. 58, No. 9, 908-910

Ziman, J.M.: Electrons and Phonons. Clarendon, Oxford (1963) 


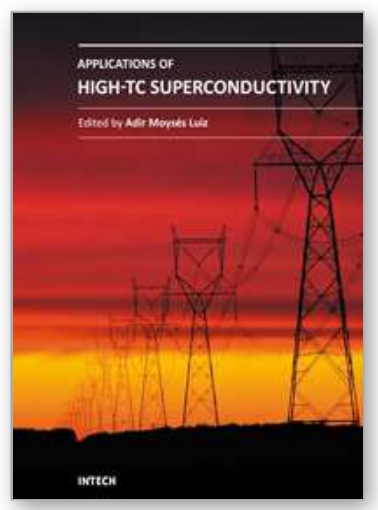

\author{
Applications of High-Tc Superconductivity \\ Edited by Dr. Adir Luiz
}

ISBN 978-953-307-308-8

Hard cover, 260 pages

Publisher InTech

Published online 27, June, 2011

Published in print edition June, 2011

This book is a collection of the chapters intended to study only practical applications of HTS materials. You will find here a great number of research on actual applications of HTS as well as possible future applications of HTS. Depending on the strength of the applied magnetic field, applications of HTS may be divided in two groups: large scale applications (large magnetic fields) and small scale applications (small magnetic fields). 12 chapters in the book are fascinating studies about large scale applications as well as small scale applications of HTS. Some chapters are presenting interesting research on the synthesis of special materials that may be useful in practical applications of HTS. There are also research about properties of high-Tc superconductors and experimental research about HTS materials with potential applications. The future of practical applications of HTS materials is very exciting. I hope that this book will be useful in the research of new radical solutions for practical applications of HTS materials and that it will encourage further experimental research of HTS materials with potential technological applications.

\title{
How to reference
}

In order to correctly reference this scholarly work, feel free to copy and paste the following:

Asghari Maqsood and M. Anis-ur-Rehman (2011). Thermophysical Properties of Bi-based High-Tc Superconductors, Applications of High-Tc Superconductivity, Dr. Adir Luiz (Ed.), ISBN: 978-953-307-308-8, InTech, Available from: http://www.intechopen.com/books/applications-of-high-tcsuperconductivity/thermophysical-properties-of-bi-based-high-tc-superconductors

\section{INTECH}

open science | open minds

\section{InTech Europe}

University Campus STeP Ri

Slavka Krautzeka 83/A

51000 Rijeka, Croatia

Phone: +385 (51) 770447

Fax: +385 (51) 686166

www.intechopen.com

\section{InTech China}

Unit 405, Office Block, Hotel Equatorial Shanghai

No.65, Yan An Road (West), Shanghai, 200040, China

中国上海市延安西路65号上海国际贵都大饭店办公楼 405 单元

Phone: +86-21-62489820

Fax: $+86-21-62489821$ 
(C) 2011 The Author(s). Licensee IntechOpen. This chapter is distributed under the terms of the Creative Commons Attribution-NonCommercialShareAlike-3.0 License, which permits use, distribution and reproduction for non-commercial purposes, provided the original is properly cited and derivative works building on this content are distributed under the same license. 